TRANSACTIONS OF THE

AMERICAN MATHEMATICAL SOCIETY

Volume 352, Number 7, Pages 3323-3338

S 0002-9947(00)02483-1

Article electronically published on March 21, 2000

\title{
HYPERBOLIC MINIMIZING GEODESICS
}

\author{
DANIEL OFFIN
}

\begin{abstract}
We apply the intersection theory for Lagrangian submanifolds to obtain a Sturm type comparison theorem for linearized Hamiltonian flows. Applications to the theory of geodesics are considered, including a sufficient condition that arclength minimizing closed geodesics, for an $n$-dimensional Riemannian manifold, are hyperbolic under the geodesic flow. This partially answers a conjecture of G. D. Birkhoff.
\end{abstract}

\section{INTRODUCTION}

Let $\mathbb{X}$ denote an $n$-dimensional manifold with cotangent bundle $T^{*} \mathbb{X}$ and canonical projection $\tau: T^{*} \mathbb{X} \longrightarrow \mathbb{X}$. A famous result of Poincaré [19], [10] asserts that when $\mathbb{X}$ is an orientable surface, closed minimizing geodesics are unstable when considered as an orbit of the geodesic flow. The main theorem of this paper gives a sufficient condition, in terms of a discrete symmetry $\mathbf{R}$ on $T \mathbb{X}$, that an arclength minimizing closed geodesic on an $n$-dimensional Riemannian manifold $\mathbb{X}$ is hyperbolic under the geodesic flow in the tangent bundle. This condition partially confirms a conjecture of G. D. Birkhoff [6, p. 130], which in general is false, as attested to by a counterexample due to V. Bangert [13.

Our result is part of a larger theory of minimizing orbits of (multidimensional) Hamiltonian systems. This theory continues to play an important role in the investigation of generalizations of the Aubry-Mather theory [4], [5], [14], [15]. Properties of minimizing orbits, such as the one which we investigate here, can be crucial in establishing the existence of higher dimensional invariant Mather sets. In [18, the author investigates the condition of hyperbolicity for periodic and almost periodic minimizing orbits of planar Hamiltonian systems. Future work should extend these results to the multidimensional setting. In [17], the author investigated an earlier version of the results which appear here.

Let us establish some terminology which will be used throughout the paper. The cotangent bundle is equipped with a canonical one-form $\theta$ which, in local coordinates $\left(q_{1}, \ldots, q_{n}, p_{1}, \ldots, p_{n}\right)$, is given by

$$
\theta=\sum p_{i} d q_{i}
$$

The canonical symplectic form $\omega$ on $T^{*} \mathbb{X}$ is given by

$$
\omega=-d \theta \text {. }
$$

Received by the editors September 18, 1998.

2000 Mathematics Subject Classification. Primary 37J45, 37J50, 58E30; Secondary 53C20, 34D08, 58E10.

This research supported in part by NSERC grant OGP0041872. 
Let $H=H\left(q_{1}, \ldots, q_{n}, p_{1}, \ldots, p_{n}\right)$ denote a Hamiltonian function on $T^{*} \mathbb{X}$, and $X_{H}$ the Hamiltonian vector field defined by

$$
X_{H}=\omega^{-1} d H .
$$

We will denote the flow of $X_{H}$, which we assume to be complete, by

$$
\phi_{t}: T^{*} \mathbb{X} \longrightarrow T^{*} \mathbb{X}, \quad t \in \mathbb{R} .
$$

Our main theorem is presented as an application of an abstract comparison theorem from symplectic geometry. As alluded to above, this theorem was motivated by questions concerning the orbital stability of closed phase curves $\phi_{t} z$ of the Hamiltonian vector field, when $\tau\left(\phi_{t} z\right)$ is a minimizing curve for the associated Lagrangian action

$$
A(x)=\int L(x, \dot{x}) d t .
$$

Here $L=L(x, \dot{x})$ is the Lagrangian obtained from the Hamiltonian $H$ under the Legendre transform. Our attempts to generalize Poincaré's result hinge upon the comparison theorem which is proven in the last section.

This comparison theorem, we think, has independant interest. To support this we offer a second application of the comparison theorem. This involves a Sturmian type of comparison for Jacobi fields along a geodesic segment of a Riemannian manifold $\mathbb{X}$. Specifically, we compare the focal points of two submanifolds $M, M^{*}$, which are assumed to have a common tangent space at $q$, along a unit speed geodesic $c(t)$ through $q$ whose tangent vector $\dot{c}(0)$ is perpendicular to the common tangent space of $M, M^{*}$ at $c(0)=q$. This comparison involves the difference of the Weingarten operators $S_{\dot{c}(0)}^{*}-S_{\dot{c}(0)}$. This difference is a symmetric operator on the common tangent spaces $T_{c(0)} M^{*}$ and $T_{c(0)} M$. If this difference is positive, then our comparison theorem applies: the first focal point of $M$ occurs not before the first focal point of $M^{*}$. This statement is a strengthened form of a result by Warner [21]. In Warner's result the same conclusion holds, provided that all the eigenvalues $\mu_{1}^{*}, \ldots, \mu_{k}^{*}$ of $S_{\dot{c}(0)}^{*}$ are strictly larger than the maximum eigenvalue $\mu$ of $S_{\dot{c}(0)}$. Our condition, more general than Warner's, asks only that

$$
S_{\dot{c}(0)}^{*}>S_{\dot{c}(0)}
$$

as symmetric operators.

Now we would like to give some background and notation, from symplectic geometry, for the comparison theorem. For simplicity we denote $M=T^{*} \mathbb{X}$. An $n$-dimensional plane $\lambda$ in $T_{z} M$, for which $\left.\omega\right|_{\lambda}=0$, is called a Lagrange plane. Here and henceforth, we denote the induced tangent mapping of $\phi_{t}$ by $d_{z} \phi_{t}, \quad z \in M$.

Many dynamical questions, such as multiplicity of fixed points of $\phi_{T}$ for example, or stability of periodic orbits of $\phi_{t}$, can be related to properties of the intersection number (or the Maslov index) of the curve of Lagrange planes $d_{z} \phi_{t} \lambda$ with a fixed distribution of Lagrange planes $\alpha(z)$. One such distribution is the vertical space

$$
\begin{gathered}
\alpha(z)=\operatorname{ker} d_{z} \tau, \\
\tau: M \longrightarrow \mathbb{X} .
\end{gathered}
$$

For a trajectory $\phi_{t} z$ of the Hamiltonian vector field $X_{H}$, the number of conjugate points to $\tau(z)$ (counted with multiplicity) along the trajectory $\tau\left(\phi_{t} z\right), 0 \leq t \leq T$, 
is given by the formula

$$
\text { conjugate index }=\sum_{0<t \leq T} \operatorname{dim}\left[d_{z} \phi_{t} \alpha(z) \cap \alpha\left(\phi_{t} z\right)\right] .
$$

A different (but related) example is furnished by the Maslov index of a closed curve $\gamma$ on a Lagrangian submanifold $L \subset M$. We assume that $L$ is invariant under the flow $\phi_{t}$. The Maslov index (Arnold 2]) is the algebraic intersection number of the curve $\gamma$ with the singular cycle on $L$, denoted by $\Sigma_{\alpha}$, which in turn is defined as the locus of points on $L$ where $\tau: L \longrightarrow \mathbb{X}$ is not of full rank. The cycle $\Sigma_{\alpha}$ turns out to be a two sided surface of codimension 1 , so that it becomes necessary to speak of negative and positive intersections with $\Sigma_{\alpha}$.

The Maslov index depends only on the homology class of $\gamma$ and hence is the same as the intersection number of a closed phase curve $\phi_{t} z \subset L$, which is homologous to $\gamma$. If $\phi_{t} z$ intersects $\Sigma_{\alpha}$ only transversely and always on the same side of $\Sigma_{\alpha}$ (such a curve is called a plus curve, and will be shown in section 2 to hold under general hypotheses), the intersection number can be shown to be essentially the focal index along $\phi_{t} z$, which is

$$
\sum_{0<t \leq T} \operatorname{dim}\left[d_{z} \phi_{t}\left(T_{z} L\right) \cap \alpha\left(\phi_{t} z\right)\right] .
$$

The comparison theorem we present in this paper allows one (in certain circumstances detailed below) to deduce that the focal index of $\lambda$ is zero, if we have another Lagrange plane $\lambda^{*}$ with which to compare. The comparison is described in terms of a coordinate mapping $Q$, introduced by Duistermaat [9], on the Lagrangian Grassmanian of $\mathbb{R}^{2 n}$, with the global coordinates $\left(x_{1}, \ldots, x_{n}, y_{1}, \ldots, y_{n}\right)$ and the canonical symplectic form

$$
\omega=\sum d x_{i} \wedge d y_{i}
$$

The comparison theorem asserts that if $X_{H}$ is a Hamiltonian vector field with flow $\phi_{t}$ and $d_{z} \phi_{t} \lambda^{*}$ is a curve of Lagrange planes which does not intersect the vertical distribution $\alpha(z)$ along $\phi_{t} z, \quad 0 \leq t \leq b$, then the curve of Lagrange planes $d_{z} \phi_{t} \lambda$ also does not intersect $\alpha$ along $\phi_{t} z$, provided

A1) The Hamiltonian $H$ satisfies $\partial^{2} / H, \partial p^{2}>0$,

A2) $Q\left(\lambda, \lambda^{*} ; \alpha\right) \geq 0$,

A3) $\lambda \cap \alpha(z)=\{0\}$.

The first condition is a general one which guarantees the positivity of the intersection discussed above. The second and third conditions form the basis of the comparison between the two Lagrange planes $\lambda, \lambda^{*}$.

We begin by collecting some information and terminology which will be used throughout the paper.

\section{Preliminaries}

Henceforth we will denote the Lagrangian Grassmanian of $\mathbb{R}^{2 n}$, equipped with the canonical symplectic form

$$
\omega=\sum d x_{i} \wedge d y_{i}
$$

by $\Lambda(n)$. This is the topological space of Lagrange planes in $\mathbb{R}^{2 n}$. The space $\Lambda(n)$ has a natural manifold structure, and we will use a coordinate mapping, introduced by Duistermaat [9], which we describe (for completeness) in some detail. 
A coordinate neighbourhood of a fixed Lagrange plane $\gamma \in \Lambda(n)$ is the open set $\Lambda^{0}(\gamma)$, consisting of all Lagrange planes $\beta$ with $\beta$ transverse to $\gamma$, or $\beta \cap \gamma=0$. If $\lambda, \lambda^{*} \in \Lambda^{0}(\gamma)$, we may represent $\lambda^{*}$ uniquely by summands in $\lambda$ and $\gamma$. Specifically, if $w \in \lambda^{*}, w=u+v$ with $u \in \lambda, v \in \gamma$, then $C u=v$. In other words, there is a unique linear transformation

$$
C: \lambda \longrightarrow \gamma
$$

such that

$$
\lambda^{*}=\{u+C u \mid u \in \lambda\} .
$$

We will refer to the representation (11) with the shorthand $\lambda^{*}=\operatorname{graph} C$. A useful observation is that

$$
\text { kernel } C=\lambda \cap \lambda^{*} \text {. }
$$

Now, following Duistermaat, we define the bilinear form $Q$ on $\lambda$, which is a coordinate mapping on $\Lambda^{0}(\gamma)$,

$$
Q=Q\left(\lambda, \gamma ; \lambda^{*}\right)\left(u_{0}, u_{1}\right)=\omega\left(C u_{0}, u_{1}\right),
$$

where $u_{i} \in \lambda$ and $\omega$ is the canonical symplectic form on $\mathbb{R}^{2 n}$. It is a simple calculation to show that $Q$ is a symmetric bilinear form on $\lambda$. If $w_{i}=u_{i}+v_{i}, i=0,1$, where $u_{i} \in \lambda$ and $v_{i} \in \gamma$, then

$$
\begin{aligned}
\omega\left(C u_{0}, u_{1}\right) & =\omega\left(v_{0}, u_{1}\right) \\
& =\omega\left(u_{0}+v_{0}, u_{1}\right) \\
& =\omega\left(u_{0}+v_{0},-v_{1}\right) \\
& =\omega\left(v_{1}, u_{0}\right) \\
& =\omega\left(C u_{1}, u_{0}\right) .
\end{aligned}
$$

The coordinate mapping $Q$ reduces many geometrical questions concerning $\lambda \cap \lambda^{*}$ to evaluations using the signature of $Q$. We mention here the local formula for the Maslov index of a generic curve $\lambda_{t}$ of Lagrange planes, relative to the Lagrange plane $\alpha$ :

$$
\left[\lambda_{t} ; \alpha\right]=\frac{1}{2} \sum_{0<t \leq T} \triangle \operatorname{sgn} Q\left(\lambda_{t}, \beta ; \alpha\right) .
$$

Here the sum is taken over those $t$ with $\lambda_{t} \cap \alpha \neq 0$. By generic we mean that the curve $\lambda_{t}$ intersects the variety (Maslov cycle) $\Sigma_{\alpha}$ transversely. For a smooth generic curve $\lambda_{t}$, the tangent direction $\frac{d}{d t} \lambda_{t}$ is transverse to $\Sigma_{\alpha}$, that is

$$
\text { nullspace }\left.\left\{\frac{d}{d t} Q\left(\lambda_{t}, \beta ; \alpha\right)\right\}\right|_{\lambda_{t} \cap \alpha}=\{0\} .
$$

We suggest the following considerations to explain the formula (4). Intersections of $\lambda_{t}$ and $\alpha$ correspond to zero eigenvalues of the form $Q\left(\lambda_{t}, \beta ; \alpha\right)$, according to equation (2). If $\lambda_{t}$ intersects $\Sigma_{\alpha}$ transversely, then the signature of $Q\left(\lambda_{t}, \beta ; \alpha\right)$ changes by \pm 2 , depending on whether the eigenvalue goes through 0 from negative to positive (corresponding to $\triangle \operatorname{sgn} Q\left(\lambda_{t}, \beta ; \alpha\right)=2$ ) or vice versa (corresponding to $\left.\triangle \operatorname{sgn} Q\left(\lambda_{t}, \beta ; \alpha\right)=-2\right)$.

There are two very useful properties of the coordinate mapping $Q(\lambda, \beta ; \alpha)$ which we will need later. For proofs of these properties, see Duistermaat [9]. The first 
one is a cyclic property of $\mathrm{Q}$ which holds when $\lambda \cap \alpha=\{0\}$ in addition to $\lambda \cap \beta=$ $\alpha \cap \beta=0$. In this case (using $\approx$ to denote similarity of quadratic forms),

$$
Q(\lambda, \beta ; \alpha) \approx Q(\alpha, \lambda ; \beta) .
$$

The second property is that for any (linear) symplectic transformation $f$,

$$
Q(\lambda, \beta ; \alpha) \approx Q(f \lambda, f \beta ; f \alpha) .
$$

We have now come to the point where we can describe the condition that $\lambda_{t}$ be a plus curve. Loosely speaking, we want to ensure that $\lambda_{t}$ crosses $\Sigma_{\alpha}$ transversely and with only a positive contribution to the Maslov index $\left[\lambda_{t} ; \alpha\right]$; see equation (4). More precisely, if we let $\rho_{\lambda \cap \alpha}$ denote the restriction map to the subspace $\lambda \cap \alpha$, for the quadratic form $Q(\lambda, \beta ; \alpha)$, we will need to evaluate the restriction of the tangent vector

$$
\rho_{\lambda \cap \alpha} \frac{d}{d t} Q(\lambda, \beta ; \alpha)
$$

where $\lambda=\lambda_{t}$.

Definition 2.1. A smooth curve of Lagrange planes $\lambda_{t}$ is a plus curve (relative to the Lagrange plane $\alpha$ ) if

$$
\rho_{\lambda \cap \alpha} \frac{d}{d t} Q\left(\lambda_{t}, \beta ; \alpha\right)>0
$$

where $\lambda=\lambda_{t}$ and $\lambda_{t} \cap \alpha \neq 0$.

We remind the reader that by convention we denote the vertical distribution by $\alpha=\alpha(z)$.

Lemma 2.1. Suppose the Hamiltonian $H$ satisfies the condition A1). Then the curve of Lagrange planes $\gamma_{t}=d \phi_{-t} \alpha$ is a plus curve; that is, if $\gamma_{t} \subset \Lambda^{0}\left(\lambda^{*}\right)$, then

$$
\rho_{\gamma_{t} \cap \lambda} \frac{d}{d t} Q\left(\gamma_{t}, \lambda^{*} ; \lambda\right)>0 .
$$

Proof. We start with the similarity transformation

$$
Q\left(\gamma_{t}, \lambda^{*} ; \lambda\right) \cong Q\left(\alpha, d \phi_{t} \lambda^{*} ; d \phi_{t} \lambda\right)
$$

Choosing the global coordinate system $\left(x_{1}, \ldots, x_{n}, y_{1}, \ldots, y_{n}\right)$ in $\mathbb{R}^{2 n}$, we identify the linearized flow mapping $d \phi_{t}$ with the fundamental solution for the linear Hamiltonian system

$$
\begin{gathered}
x^{\prime}=B(t) x+C(t) y, \\
y^{\prime}=-A(t) x+B^{T}(t) y,
\end{gathered}
$$

where the Hessian of $H$ is the $2 n \times 2 n$ matrix

$$
H_{z z}\left(d \phi_{t} z\right)=\left[\begin{array}{ll}
A(t) & B^{T}(t) \\
B(t) & C(t)
\end{array}\right]
$$

with $A, C$ symmetric $n \times n$ matrices and $B^{T}$ the transpose of $B$. Now let $(x, y) \in \lambda$ and $(\xi, \eta) \in \lambda^{*}$. We also denote

$$
\begin{aligned}
& (x(t), y(t))=d \phi_{t}(x, y), \\
& (\xi(t), \eta(t))=d \phi_{t}(\xi, \eta) .
\end{aligned}
$$

We may assume that $d \phi_{t} \lambda^{*} \cap \alpha(z)=\{0\}$, which implies that if $\xi(t)=0$ for some $t$, then $\eta(t)=0$ as well. 
Now in order to explicitly calculate the quadratic form $Q\left(\alpha, d \phi_{t} \lambda^{*} ; d \phi_{t} \lambda\right)$, we need to describe the linear mapping

$$
C: \quad \alpha \longrightarrow d \phi_{t} \lambda^{*}
$$

Since $\xi(t) \neq 0$ for each $t$, when $(\xi, \eta) \neq 0$, suppose that $x(t)=\xi(t)$, where $(x, y) \in \lambda$. We can obviously write, for this value of $t$,

$$
\left(\begin{array}{l}
x(t) \\
y(t)
\end{array}\right)=\left(\begin{array}{l}
\xi(t) \\
\eta(t)
\end{array}\right)+\left(\begin{array}{c}
0 \\
y(t)-\eta(t)
\end{array}\right) .
$$

It is only necessary to recognize this as the explicit representation $d \phi_{t} \lambda=\operatorname{graph} C$; see equation (1).

To be precise, we need to check that

$$
\left(\begin{array}{c}
0 \\
y(t)-\eta(t)
\end{array}\right) \longrightarrow\left(\begin{array}{c}
\xi(t) \\
\eta(t)
\end{array}\right)
$$

is a well defined linear mapping from $\alpha$ to $d \phi_{t} \lambda^{*}$. Since we have chosen $\lambda^{*}$ with

$$
\lambda \cap \lambda^{*}=\{0\},
$$

it follows from (8) that $y(t)-\eta(t) \neq 0$ for any nonzero choice of $(x, y) \in \lambda$. In other words, this condition guarantees that every element of $\alpha$ may be represented as the coordinate vector $\left(\begin{array}{c}0 \\ y(t)-\eta(t)\end{array}\right)$, for some choice of $\left(\begin{array}{l}x \\ y\end{array}\right) \in \lambda$. The mapping $C$, described by equation (9), is certainly linear, and thus equation (8) may be recast as

$$
d \phi_{t} \lambda=\alpha+C \alpha
$$

Having the linear map $C$ at our disposal allows us to calculate the quadratic form $Q=Q\left(\alpha, d \phi_{t} \lambda^{*} ; d \phi_{t} \lambda\right)$ (see equation (3) ). If we denote (for the sake of convenience) $v=y(t)-\eta(t)$, we deduce, using equation (8) together with $x(t)=\xi(t)$, that

$$
Q\left(\left(\begin{array}{l}
0 \\
v
\end{array}\right),\left(\begin{array}{l}
0 \\
v
\end{array}\right)\right)=\omega\left(C\left(\begin{array}{l}
0 \\
v
\end{array}\right),\left(\begin{array}{l}
0 \\
v
\end{array}\right)\right)=\langle x(t), y(t)-\eta(t)\rangle,
$$

where we have used $\langle\cdot, \cdot\rangle$ to denote the standard inner product in $\mathbb{R}^{n}$.

We may use equation (10) to test the statement of equation (7) in Lemma 2.1. Specifically, we assume that $d \phi_{-t} \alpha \cap \lambda \neq\{0\}$. This means that there is some $(x, y) \in \lambda$ with $x(t)=0$. We now compute

$$
\begin{aligned}
\frac{d}{d t} Q\left[\left(\begin{array}{l}
0 \\
v
\end{array}\right),\left(\begin{array}{l}
0 \\
v
\end{array}\right)\right] & =\left\langle x^{\prime}(t), y(t)-\eta(t)\right\rangle+\left\langle x(t), y^{\prime}(t)-\eta^{\prime}(t)\right\rangle \\
& =\langle C(t) y(t), y(t)\rangle
\end{aligned}
$$

where we have used the fact that $x(t)=0$ and that $y(t) \neq 0$, because $(x, y) \neq 0$. Since $C(t)>0$ due to the assumption A1), condition (7) follows from our previous calculation. This completes the proof.

We remark that the conclusion of the theorem applies even when $\lambda \cap \lambda^{*} \neq 0$, provided that $\gamma_{t} \subset \Lambda^{0}\left(\lambda^{*}\right)$. 


\section{Symmetric Minimizing GeOdesics}

Our main application concerns the variational theory for closed geodesics on a Riemannian manifold $\mathbb{X}$. Recall that for the family of curves $C:[0,1] \rightarrow \mathbb{X}$ which satisfy the periodicity condition $c(0)=c(1)$, we define the energy integral $E$ by

$$
E(c)=\frac{1}{2} \int_{0}^{1}\langle\dot{c}, \dot{c}\rangle d t .
$$

$E$ is stationary if and only if $c(t), 0 \leq t \leq 1$, is a closed geodesic with respect to the metric $\langle\cdot, \cdot\rangle$. In this and the following, by curve we will mean a piecewise smooth map from $[0,1]$ to $\mathbb{X}$. It is well known that if $\mathbb{X}$ is compact, then every nontrivial homotopy class of closed curves on $\mathbb{X}$ has as representative a closed geodesic, which minimizes $E$ over that class. The mere existence of such a geodesic, however, does not imply anything about the dynamic character of the geodesic flow.

The dynamical aspect of this theory was brought out by Poincaré, who showed that if $\mathbb{X}$ is an oriented 2-manifold, then every minimal closed geodesic is hyperbolic, which means that the linearized Poincaré map has all eigenvalues off the unit circle in the complex plane. This fact turns out to have profound implications for the existence of other periodic geodesics which are in general not minimizing; see for example Bangert 3]. G. D. Birkhoff 6] (p. 130) conjectured that Poincaré's result generalized to higher dimensions. The following construction comprises a counterexample to Birkhoff's conjecture (due to V. Bangert [13]). Let $\mathbb{X}=\mathbb{R}^{3} / \sim$, where the equivalence relation $\sim$ identifies the two planes $z=0$ and $z=1$, when $m=(x, y, z)$ belongs to $\mathbb{X}$. The metric on $\mathbb{X}$ is written in cylindrical coordinates $(r, \theta, z)(z \bmod 1)$ as

$$
d s^{2}=d r^{2}+r^{2} d(\theta-\alpha z)^{2}+d z^{2}
$$

where $\alpha$ is a fixed parameter, $0 \leq \alpha \leq \pi$. Observe that

$$
r=0, \quad z=t, \quad 0 \leq t \leq 1,
$$

describes a minimal closed geodesic. However, the linearized Poincaré map has repeated eigenvalues on the unit circle. In this case, the geodesic is called elliptic.

We shall describe a situation under which a minimizing geodesic on an $n$-dimensional manifold $\mathbb{X}$ is hyperbolic. Let $\mathbb{X}$ denote a Riemannian manifold with tangent bundle $T \mathbb{X}$. The Riemannian metric $g$ on $\mathbb{X}$ induces an isomorphism between $T \mathbb{X}$ and $T^{*} \mathbb{X}$. Define a symplectic form on $T \mathbb{X}$ by pulling back the canonical symplectic form on $T^{*} \mathbb{X}$ via this isomorphism. Denote this pulled-back form by $\omega$.

The metric $g$ also defines a connection and, therefore, a splitting of $T(T \mathbb{X})$ into horizontal and vertical spaces (see for example [12], pp. 47, 48), which we will denote by

$$
T_{z}(T \mathbb{X})=T_{z h}(T \mathbb{X})+T_{z v}(T \mathbb{X})
$$

Each of the horizontal and vertical spaces is canonically isomorphic to $T_{x} \mathbb{X}$, where $\tau z=x(\tau$ is the projection $\tau: T \mathbb{X} \longrightarrow \mathbb{X})$. The symplectic form acts on $T_{z}(T \mathbb{X})$ as follows. Let $\xi=\left((\xi)_{h}+(\xi)_{v}\right) \in T_{z}(T \mathbb{X})$, where the subscripts denote the projections into the horizontal and vertical spaces. Then

$$
\omega\left(\xi_{1}, \xi_{2}\right)=\left\langle\left(\xi_{1}\right)_{h},\left(\xi_{2}\right)_{v}\right\rangle-\left\langle\left(\xi_{1}\right)_{v},\left(\xi_{2}\right)_{h}\right\rangle,
$$

where $\langle$,$\rangle denotes the Riemannian scalar product on T_{x} \mathbb{X}$ (see [12], Proposition 3.1.14).

We assume that $T \mathbb{X}$ admits an antisymplectic, isometric involution $\mathbf{R}$ and that there is a submanifold $Q$ of $\mathbb{X}$ such that the fixed point set of $\mathbf{R}$, denoted $\operatorname{Fix}(\mathbf{R})$, 
is the normal bundle of $Q, \operatorname{Fix}(\mathbf{R})=N(Q)$. A mapping $\mathbf{R}: T \mathbb{X} \longrightarrow T \mathbb{X}$ is an antisymplectic, isometric involution under the following conditions:

$$
\mathbf{R}^{2}=\mathrm{id}, \quad \mathbf{R}^{*} \omega=-\omega, \quad \text { and } \mathbf{R} \text { is isometric on the fibres. }
$$

Conditions (11) guarantee that the vector field $G$ on $T \mathbb{X}$, which generates the geodesic flow, is reversed under this map [16]:

$$
\mathbf{R}_{*} G=-G \circ \mathbf{R} .
$$

We will henceforth assume that $\operatorname{Fix}(\mathbf{R})=N(Q) \cup N(K)$, where $Q$ and $K$ denote submanifolds of $\mathbb{X}$. Under these conditions we consider the following variational problem:

$$
\text { minimize } E(c) \text { over all curves which join } Q \text { and } K \text {. }
$$

We make the observation that the conditions (11) also imply that the $\mathbb{Z}_{2}$ symmetry $\dot{c} \rightarrow \mathbf{R} \dot{c}$ leaves the functional $E$ invariant.

If $\underline{c}$ is a solution to the variational problem [13), then by transversality, $\underline{\dot{c}}(0) \in$ $N(Q)$ and $\underline{\dot{c}}(1) \in N(K)$. Moreover, since $\operatorname{Fix}(\mathbf{R})=N(Q) \cup N(K)$, it follows from (12) that $\underline{c}$ may be continued smoothly to a closed geodesic on the doubled interval $0 \leq t \leq 2$ (see 16] for a proof). Thus, solutions of the variational problem yield $\mathbf{R}$-symmetric closed geodesics. Before we prove that such geodesics are hyperbolic, we will consider some illustrative examples.

An example on $S^{2}$ from Devaney [8] starts with the map $\mathbf{S}$ on $\mathbb{R}^{3}$ which is a reflection through a plane containing the origin. We denote the restriction to $S^{2}$ also by $\mathbf{S}$. Since $\mathbf{S}$ is symmetric and involutive, $\mathbf{S}_{*}: T S^{2} \longrightarrow T S^{2}$ is a symplectic map with standard symplectic form restricted to $T S^{2}$, which is isometric on the fibres of $T S^{2}$. Finally, $\mathbf{R}=-\mathbf{S}_{*}$ is an antisymplectic isometric involution whose fixed point set is $N(Q)$, where $Q$ is a great circle on $S^{2}$. This example has infinitely many symmetric closed geodesics (a geodesic $c(\cdot)$ is called symmetric if $(c(0), \dot{c}(0)$ ) belongs to $\operatorname{Fix}(\mathbf{R})$ ), none of which are minimal however. The same construction for the standard two-torus embedded in $\mathbb{R}^{3}$ gives either infinitely many (degenerate) minimal symmetric geodesics or an isolated (nondegenerate) one, depending on which reflection is used. Other examples of such discrete symmetries occuring in dynamics may be found in Devaney [8] or Meyer [16].

An example using the same construction as above to produce a hyperbolic, symmetric, $E$-minimizing geodesic on $\mathbb{X}=S^{2} \times S^{1}$ is as follows. We take local coordinates $(\theta, \phi)$ on $S^{2}$, and $x(\bmod 2)$ as coordinate on $S^{1}$. The metric in these coordinates is

$$
d s^{2}=\left(1+\cos ^{2} \phi+\sin ^{2} \theta\right) d x^{2}+\sin ^{2} \phi d \theta^{2}+d \phi^{2} .
$$

It is easy to see that the geodesic $\underline{c}$ defined by $x=t, \theta=0, \phi=\frac{\pi}{2}, \quad 0 \leq t \leq 2$, is a closed, $E$-minimizing geodesic which is also hyperbolic. The sectional curvatures along $\underline{c}$ are -1 and -1 , which gives a different confirmation of the hyperbolicity of c.

We assert that $\underline{c}$ is a symmetric minimizing geodesic, thus falling within the category under consideration. To see this, we begin with the involution $\mathbf{S}$ on $\mathbb{X}$, $(s, x) \rightarrow(s, 2-x)$, where $s \in \mathbf{S}^{2}$. Then $\mathbf{R}=-\mathbf{S}_{*}$ is an antisymplectic, isometric involution on $T \mathbb{X}$ with $\operatorname{Fix}(\mathbf{R})=N(Q) \cup N(K)$, where $Q=S^{2} \times\{0\}$, and $K=$ $S^{2} \times\{1\}$. Moreover, $\underline{\dot{\underline{c}}}(0)$ is perpendicular to $Q$ at $\underline{c}(0)$, while $\underline{\dot{\underline{c}}}(1)$ is perpendicular to $K$ at $\underline{c}(1)$. This means that $\underline{c}$ is $\mathbf{R}$-symmetric. 
Theorem 3.1. Let $\mathbf{R}$ denote an antisymplectic, isometric involution (see (11)) and suppose that $\underline{c}$ is an $\mathbf{R}$-symmetric closed geodesic which is a solution to the variational problem (13). If the linearized Poincaré map $\Phi$ has no eigenvalues equal to +1 , then $(\underline{c}(\cdot), \underline{\dot{c}}(\cdot))$ is hyperbolic on $T \mathbb{X}$.

The proof will follow from several preliminary results. Our first task is to show that the curve of Lagrange planes $\Phi_{t} \lambda$ has no intersections with the singular cycle of the vertical field $\alpha$ over the entire evolution of the geodesic, $-\infty<t<\infty$. Here $\Phi_{t}$ denotes the linearized geodesic flow along $(\underline{c}, \underline{\dot{c}})$, and $\lambda$ is the tangent space to $N(Q)=\operatorname{Fix}(\mathbf{R})$ at $(\underline{c}, \underline{\dot{c}})(0)$. The fact that $\lambda$ is Lagrange follows from Lemma 4.1.

In order to compute the intersection number $\left[\Phi_{t} \lambda ; \alpha\right]$, we must first describe the index theorem for the second variation of $E$ along $\underline{c}$. References for this result include Ambrose [1] and Kalish [11. We will use the notation from Kalish [11. Let $S_{\underline{\dot{\underline{x}}}(0)}$ and $S_{\underline{\underline{\dot{c}}}(1)}$ denote the Weingarten operators for the second fundamental forms of the submanifolds $Q$ and $K$, relative to $\underline{\dot{c}}(0), \underline{\dot{c}}(1)$ respectively. Define the symmetric form $A$ on $\lambda$ as follows: for $z_{1}, z_{2}$ belonging to $\lambda$, let $\bar{z}_{1}=\Phi_{1} z_{1}, \bar{z}_{2}=\Phi_{1} z_{2}$; then

$$
A\left(z_{1}, z_{2}\right)=\left\langle\left(\bar{z}_{1}\right)_{v}-S_{\underline{\dot{c}}(1)}\left(\bar{z}_{1}\right)_{h},\left(\bar{z}_{2}\right)_{h}\right\rangle .
$$

Index Theorem.

$$
\operatorname{index} D^{2} E(\underline{c})=\sum_{i=1}^{k} m_{i}+\operatorname{index} A,
$$

where $m_{i}$ are the multiplicities of the $Q$-focal points along $\underline{c}(t), 0<t<1$, and the symmetric form $A$ is as defined in (14).

Theorem 3.2. If $\underline{c}$ is an $\mathbf{R}$-symmetric closed geodesic which is a solution of the variational problem (13), and $\lambda=T_{\underline{\dot{\dot{c}}(0)}} \operatorname{Fix}(\mathbf{R})$, then $\Phi_{t} \lambda$ has no intersections with the singular cycle for all real $t,-\infty<t<\infty$.

Proof. Let $\lambda=T_{\underline{\dot{c}}(0)} \operatorname{Fix}(\mathbf{R})$ and $\lambda^{*}=T_{\underline{\dot{c}}(1)} \operatorname{Fix}(\mathbf{R})$. We will apply Theorem 5.1 to the Lagrange planes $\Phi_{t} \lambda$ and $\lambda^{*}$. In order to compute the form $Q\left(\Phi_{1} \lambda, \alpha ; \lambda^{*}\right)$, we will use the following splitting formula: if $\zeta \in \Phi_{1} \lambda$ and $P$ denotes the projection of $T_{c(1)} \mathbb{X}$ onto $T_{c(1)} K$, then

$$
(\zeta)_{h}+(\zeta)_{v}+S_{\underline{\dot{c}}(1)} P(\zeta)_{h}-P(\zeta)_{v} \in \lambda^{*},
$$

while

$$
(\zeta)_{h}+(\zeta)_{v} \in \lambda
$$

and

$$
S_{\underline{\dot{c}}(1)} P(\zeta)_{h}-P(\zeta)_{v} \in \alpha
$$

For $\zeta_{1}, \zeta_{2} \in \Phi_{1} \lambda$,

$$
\begin{aligned}
Q\left(\Phi_{1} \lambda, \alpha ; \lambda^{*}\right)\left(\zeta_{1}, \zeta_{2}\right) & =\omega\left(S_{\dot{\dot{c}}(1)} P\left(\zeta_{1}\right)_{h}-P\left(\zeta_{1}\right)_{v},\left(\zeta_{2}\right)_{h}+\left(\zeta_{2}\right)_{v}\right) \\
& =\left\langle P\left(\zeta_{1}\right)_{v}-S_{\underline{\dot{ }}(1)} P\left(\zeta_{1}\right)_{h},\left(\zeta_{2}\right)_{h}\right\rangle \\
& =\left\langle\left(\zeta_{1}\right)_{v}-S_{\underline{\dot{c}}(1)} P\left(\zeta_{1}\right)_{h}, P\left(\zeta_{2}\right)_{h}\right\rangle \\
& =A\left(\zeta_{1}, \zeta_{2}\right) \geq 0 .
\end{aligned}
$$

The inequality (15) follows from equation (14) and the index theorem described above. By the same argument, along an $\mathbf{R}$-symmetric geodesic minimizing $E, \Phi_{t} \lambda$ will have no intersections with the singular cycle on the interval $0<t \leq 1$ (the 
sum of the multiplicities of $Q$-focal points is zero). By virtue of the symmetry $\mathbf{R}$, therefore,

$$
\left[\Phi_{t} \lambda^{*} ; \alpha\right]=0, \quad 0<t \leq 1 .
$$

We now wish to compare this with intersection number $\left[\Phi_{t}\left(\Phi_{1} \lambda\right) ; \alpha\right]$, which is also zero in light of (15) and Theorem 5.1 Using our earlier argument involving symmetry, we conclude that $\left[\Phi_{t} \lambda^{*} ; \alpha\right]=0$, provided that $0<t \leq 2$. This new information may be used in conjunction with our comparison argument on the interval $0<t \leq 2$, which then yields the condition that $\left[\Phi_{t}\left(\Phi_{1} \lambda\right) ; \alpha\right]=0$, provided that $0<t \leq 2$.

Thus, by successively comparing intersection numbers and extending by virtue of the symmetry $\mathbf{R}$, we conclude that

$$
\left[\Phi_{t} \lambda ; \alpha\right]=0, \quad 0<t \leq \infty .
$$

To extend this result to $\mathbb{R}$, we merely notice that the equality

$$
\mathbf{R} \Phi_{-t}=\Phi_{t} \mathbf{R}
$$

applied to $\lambda$ implies that $\left[\Phi_{t} \lambda ; \alpha\right]=0, t \varepsilon \mathbb{R} /\{0\}$.

A closed geodesic $\underline{c}$ with no conjugate points on $\mathbb{R}$ must necessarily be minimal with respect to periodic boundary conditions. This follows from a result of Bott [7] on the Morse indices of multiple covers of $\underline{c}$. If $\underline{c}_{m}$ is defined by $\underline{c}_{m}(t)=\underline{c}(t)$, on the interval $0 \leq t \leq m$, then $\underline{c}_{m}$ is called the $m$-fold multiple cover of $\underline{c}$. Here $\underline{c}_{m}$ is also a critical point of $E$ (with periodic boundary conditions $\left.\underline{c}_{m}(0)=\underline{c}_{m}(m)\right)$. Bott's result implies that if the Morse index of $D^{2} E(\underline{c})$ is positive, then $\lim _{m \longrightarrow \infty}$ index $D^{2} E\left(\underline{c}_{m}\right)=+\infty$. Since the latter condition does not occur if $\underline{c}$ has no conjugate points in $\mathbb{R}$, then necessarily, index $D^{2} E(\underline{c})=0$. Thus, we have proven the following corollary to Theorem 5.1

Corollary 3.1. An $\mathbf{R}$-symmetric closed geodesic $\underline{c}$ that minimizes the functional $E$ over the class of curves joining $Q$ and $K$, also minimizes $E$ in the free homotopy class of closed curves $\{\underline{c}\}$.

In order to discuss the hyperbolicity of $\mathbf{R}$-symmetric geodesics $\underline{c}$, we consider the linearized Poincaré map $\Phi=\Phi_{2}$ along the closed geodesic $\underline{c}$. The reversing property (12) implies that

$$
\Phi \circ \mathbf{R}_{*}=\mathbf{R}_{*} \circ \Phi^{-1} .
$$

Let $\lambda, \bar{\lambda}$ denote the eigenspaces of $\mathbf{R}_{*}$ at $\underline{\dot{c}}(0)$ which correspond to the eigenvalues $+1,-1$ respectively. Then $\lambda \cap \bar{\lambda}=0$, and $\lambda, \bar{\lambda}$ are transverse Lagrange planes. However, $\lambda, \bar{\lambda}$ are not $\Phi$-invariant. The basic theorem which describes the connection between $\Phi$-invariant subspaces and the subspaces $\lambda, \bar{\lambda}$ is given by Devaney [8]. His result implies that to study the eigenvalues of the Poincaré map $\Phi$, it suffices to study the pseudo-Poincaré map ( $\Phi+\Phi^{-1}$ ), restricted to the invariant subspace $\lambda$. Since the statement we need is slightly different than the one found in Devaney, we include the proof.

Theorem 3.3 (Devaney). If

$$
\Phi \zeta=\mu \zeta \text { with } \mu \in \mathbb{C},
$$

and

$$
\zeta=v+\bar{v} \quad \text { with } \quad v \in \lambda, \bar{v} \in \bar{\lambda}
$$


then

and

$$
\left(\Phi+\Phi^{-1}\right) v=\left(\mu+\mu^{-1}\right) v
$$

$$
\left(\Phi+\Phi^{-1}\right) \bar{v}=\left(\mu+\mu^{-1}\right) \bar{v}
$$

Proof. Let $\zeta=v+\bar{v}, \quad v \in \lambda, \bar{v} \in \bar{\lambda}$, where we assume that $\lambda$ and $\bar{\lambda}$ are complexified by considering arbitrary complex multiples of elements of $\lambda, \bar{\lambda}$ respectively. Since $\lambda, \bar{\lambda}$ are transverse, we can uniquely write

$$
\Phi v=a+b, \quad \Phi \bar{v}=\bar{a}+\bar{b}
$$

with $a, \bar{a} \in \lambda$ and $b, \bar{b} \in \bar{\lambda}$. It follows that $(a+\bar{a})=\mu v$ and $b+\bar{b}=\mu \bar{v}$. On the other hand, we can use (16) to conclude that

$$
\Phi^{-1} v=\mathbf{R}_{*} \Phi v=a-b
$$

and

Therefore, since

$$
\Phi^{-1} \bar{v}=-\mathbf{R}_{*} \Phi \bar{v}=-\bar{a}+\bar{b} .
$$

$$
\Phi^{-1}(v+\bar{v})=\mu^{-1}(v+\bar{v})
$$

and

it follows that

$$
a-\bar{a}=\mu^{-1} v, \quad-b+\bar{b}=\mu^{-1} \bar{v},
$$

and

$$
\left(\Phi+\Phi^{-1}\right) v=2 a=\left(\mu+\mu^{-1}\right) v
$$

$$
\left(\Phi+\Phi^{-1}\right) \bar{v}=2 \bar{b}=\left(\mu+\mu^{-1}\right) \bar{v} .
$$

Using Devaney's theorem, it is easy to prove the following.

Corollary 3.2. Let $\underline{c}$ denote an $\mathbf{R}$-symmetric geodesic (not necessarily E-minimizing) and $\Phi$ the linearized Poincaré mapping along $\underline{c}$. If $\Phi \zeta=\mu \zeta$, with $\mu \in S^{1}$, then it is possible to choose $v, \bar{v}$, with $v \in \lambda$ and $\bar{v} \in \bar{\lambda}$, such that $v$ is strictly real and $\zeta=v+\bar{v}$.

We are now in a position to complete the proof of Theorem 3.1. We will show how the statement of Theorem 3.2 , the null rotation of the Lagrange plane $\lambda=\operatorname{Fix}(\mathbf{R})$ under the linearized flow $\Phi_{t}$, restricts the eigenvalues $\mu$ of the linearized Poincaré map $\Phi$. In particular, we will see that this condition is incompatible with the assumption $\mu \in S^{1}$ by virtue of Corollary 3.2.

Specifically, assume that

$$
\Phi(v+\bar{v})=\mu(v+\bar{v}), \quad \mu=e^{i \alpha T}, \quad 0<\alpha<\pi,
$$

where $v \in \lambda, \bar{v} \in \bar{\lambda}$, with $v^{*}=v$ (* denotes complex conjugation) by virtue of Corollary [3.2. Applying the involution $\mathbf{R}_{*}$ to $\Phi^{-1}(v+\bar{v})$ and using equation (16), we have

$$
\Phi(v-\bar{v})=\mu^{-1}(v-\bar{v}) .
$$

On the other hand, from equation (17) we compute

$$
\Phi\left(v+\bar{v}^{*}\right)=\mu^{-1}\left(v+\bar{v}^{*}\right) .
$$

Taking differences of these last two relations, we get

$$
\Phi\left(\bar{v}+\bar{v}^{*}\right)=\mu^{-1}\left(\bar{v}+\bar{v}^{*}\right)
$$


which implies that $\bar{v}+\bar{v}^{*}=0$. Therefore, since $v^{*}=v$, from (17)

$$
\begin{aligned}
& \Phi^{k} v=\cos (\alpha k T) v-\sin (\alpha k T) \bar{v}, \\
& \Phi^{k} \bar{v}=\sin (\alpha k T) v+\cos (\alpha k T) \bar{v} .
\end{aligned}
$$

These relations imply that there is a nontrivial rotation between the two Lagrange planes $\lambda, \lambda^{*}$.

In particular, $\omega\left(\bar{v}, \Phi^{k} v\right)=\cos (\alpha k T) \omega(\bar{v}, v)$, which changes sign infinitely many times as $k \rightarrow \infty$. This implies that $\left[\phi_{t} \lambda ; \beta\right]$ tends to infinity as $t \rightarrow \infty$ for some Lagrange plane $\beta$, and hence that $\left[\phi_{t} \lambda ; \nu\right]=\left[\phi_{t} \lambda ; \beta\right]+$ fixed integer (see Duistermaat 9] Theorem 2.9) also tends to infinity. This contradicts the conclusion of Theorem 3.2 and finishes the proof of Theorem 3.1 .

\section{Further Applications}

Our Theorem 5.1 is related to a classical theorem of Sturm, which compares the solutions of the two scalar second order equations

$$
\begin{array}{lll}
x^{\prime \prime}(t)+K(t) x(t)=0, & x(0)=0, & x^{\prime}(0)>0, \\
\tilde{x}^{\prime \prime}(t)+\tilde{K}(t) \tilde{x}(t)=0, & \tilde{x}(0)=0, & \tilde{x}^{\prime}(0)>0,
\end{array}
$$

when $K(t) \leq \tilde{K}(t)$. Under the assumption of this inequality, Sturm's theorem asserts that if $\tilde{x}^{\prime}(0)=x^{\prime}(0)>0$ and $\tilde{x}(t) \neq 0$ on the interval $(0, b]$, then $x(t) \geq \tilde{x}(t)$ on the interval $[0, b]$. In particular, $x(t) \neq 0$ on $(0, b]$. Here $n=1$, so all onedimensional subspaces are Lagrangian. The condition that $\tilde{x}(t) \neq 0$ corresponds to our assumption that $\left[d \phi_{t} \lambda^{*} ; \alpha\right]=0$ on $(0, b]$.

Sturm's theorem applies immediately to give a metric comparison theorem for Jacobi fields along geodesic segments of two surfaces $M$ and $M^{*}$ whose Gaussian curvatures satisfy the inequality $K_{M^{*}} \geq K_{M}$. This result was generalized by Rauch [20] to the case of $n$-dimensional Riemannian manifolds $M, M^{*}$ and corresponding geodesic segments $\gamma:[0, b] \longrightarrow M, \gamma^{*}:[0, b] \longrightarrow M^{*}$. The inequality concerning the Gaussian curvature is now replaced by families of inequalities involving sectional curvature,

$$
K^{*}\left(v^{*}, \dot{\gamma}^{*}\right) \geq K(v, \dot{\gamma})
$$

for every $v^{*} \in T_{x} M^{*}$ and $v \in T_{x} M$. The conclusion remains the same, namely, that for corresponding Jacobi fields $J^{*}, J$ along $\gamma^{*}, \gamma$ respectively,

$$
\left\|J_{(t)}\right\| \geq\left\|J_{(t)}^{*}\right\|, \quad t \in(0, b] .
$$

An extension of the Rauch comparison theorem for Jacobi fields, which satisfies more general boundary conditions, is due to Warner [21].

Our Theorem 5.1 gives a similar extension of the Rauch comparison theorem, which may be used to compare focal points of two submanifolds $M, M^{*}$ along their common orthogonal geodesic $\gamma(t)$. However, in distinction from Warner's result, we do not need such a strong inequality on the normal curvatures of the submanifolds $M, M^{*}$. The reason for this state of affairs is that, while Warner's theorem is a metric comparison between Jacobi fields, our result is a topological comparison.

We shall use the same setup here which was used in the last section. Let $\mathbb{X}$ denote a Riemannian manifold with tangent bundle $T \mathbb{X}$. The symplectic form on $T \mathbb{X}$ is described in Section 3 together with the vertical and horizontal subspaces.

Now let $M$ denote a codimension $k$ submanifold of $\mathbb{X}, m \in M$, and $p \perp T_{m} M$. The subspace of $T_{z}(T \mathbb{X})$ such that $(\xi)_{h} \in T_{m} M$ and $(\xi)_{v} \in T_{m} M$ is a symplectic subspace (with $\operatorname{dim}=2 n-2 k)$ of $T_{z}(T \mathbb{X})$. We will refer to this subspace as the 
tangential subspace (to $M)$ of $T_{z}(T \mathbb{X})$. The tangential component of the vertical vector $(\xi)_{v}$, where $\xi \in T_{z}(T \mathbb{X})$, is equal to the vector $S_{p}(\xi)_{h}$, where $S_{p}$ denotes the Weingarten operator relative to $p \perp T_{m} M$, where

$$
(\xi)_{v}=S_{p}(\xi)_{h}+\eta
$$

and $\eta$ belongs to the normal space of $T_{m} M$ (see [12], pp. 90, 92).

Let $M \subset \mathbb{X}$ denote a codimension $k$ submanifold, and $N(M)$ the normal bundle.

Lemma 4.1. $N(M)$ is a Lagrangian submanifold of $T \mathbb{X}$. If $\lambda=T_{z} N \cap$ (tangential subspace), then $\lambda$ intersects the vertical subspace trivially.

Proof. Let $\xi=\left((\xi)_{h}+(\xi)_{v}\right) \in T_{z} N$. Then $(\xi)_{h} \in T_{m} M(m=\tau z)$ and $(\xi)_{v}=$ $S_{p}(\xi)_{h}$. Therefore,

$$
\begin{aligned}
\omega\left(\xi_{1}, \xi_{2}\right) & =\left\langle\left(\xi_{1}\right)_{h},\left(\xi_{2}\right)_{v}\right\rangle-\left\langle\left(\xi_{1}\right)_{v},\left(\xi_{2}\right)_{h}\right\rangle \\
& =\left\langle S_{p}\left(\xi_{2}\right)_{h},\left(\xi_{1}\right)_{h}\right\rangle-\left\langle\left(\xi_{2}\right)_{h}, S_{p}\left(\xi_{1}\right)_{h}\right\rangle \\
& =0
\end{aligned}
$$

by symmetry of the Weingarten operator. On the other hand, $\operatorname{dim} N=n$, which shows that $N$ is a Lagrangian submanifold of $T \mathbb{X}$. Now the projection of $\lambda$ onto the horizontal subspace equals $T_{m} M$, which has dimension $n-k$. Therefore, as a Lagrangian subspace of the tangential component of $T_{z} N, \lambda$ intersects the vertical space trivially.

To apply Theorem 5.1 in this setting, let $M, M^{*}$ denote hypersurfaces with a common tangent plane at $m=c(0)$, where $c(t)$ is a (unit speed) geodesic orthogonal to both $M$ and $M^{*}$. Let $\lambda_{M}$ and $\lambda_{M^{*}}$ denote the respective tangential components of tangent spaces of normal bundles $N(M), N\left(M^{*}\right)$ at $c(0)$. Let $d \phi_{t}$ denote the linearized geodesic flow along $c(t)$.

The nontrivial intersections of $d \phi_{t} \lambda$ with the vertical space correspond to socalled focal points of the hypersurface $M$ along the geodesic $c(t)$. To compare focal points of $M$ and $M^{*}$ along $c(t)$, we must compute $Q\left(\lambda, V ; \lambda^{*}\right)$.

For this purpose, notice that if $\xi \in \lambda$, we can choose $\xi^{*} \in \lambda^{*}$ so that $(\xi)_{h}=$ $\left(\xi^{*}\right)_{h}$, since $\lambda \cap V=\lambda^{*} \cap V$. In this case,

$$
\xi^{*}=\xi+\left(\xi^{*}\right)_{v}-(\xi)_{v}
$$

Therefore, if $\xi_{1}, \xi_{2} \in \lambda$, then

$$
\begin{aligned}
Q\left(\lambda, V ; \lambda^{*}\right)\left(\xi_{1}, \xi_{2}\right) & =\omega\left(\left(\xi_{1}^{*}\right)_{v}-\left(\xi_{1}\right)_{v}, \xi_{2}\right) \\
& =-\left\langle\left(\xi_{1}^{*}\right)_{v}-\left(\xi_{1}\right)_{v},\left(\xi_{2}\right)_{h}\right\rangle \\
& =\left\langle\left(\xi_{1}\right)_{v}-\left(\xi_{1}^{*}\right)_{v},\left(\xi_{2}\right)_{h}\right\rangle .
\end{aligned}
$$

Using the Weingarten formula we discussed earlier, we observe that $\left\langle\left(\xi_{1}\right)_{v},\left(\xi_{1}\right)_{h}\right\rangle$ is the normal curvature of the hypersurface $M$ in the direction $\left(\xi_{1}\right)_{h}$. Therefore, $Q\left(\lambda, V ; \lambda^{*}\right) \geq 0$ if and only if the normal curvature of $M$ is larger than the normal curvature of $M^{*}$ in the common $(\xi)_{h} \in T_{c(0)} M$.

Applying Theorem (5.1), we conclude that if $M^{*}$ has no focal points along $c(t)$, $0 \leq t \leq \tau$, and the normal curvature of $M$ is larger than that of $M^{*}$ along every direction in $T_{c(0)} M$, then $M$ has no focal points along $c(t), 0 \leq t \leq \tau$. This may be compared with Warner [21]. 


\section{The Comparison Theorem}

To set the stage for our comparison theorem, we note that the general case of the symplectic manifold $M=T^{*} \mathbb{X}$, with canonical symplectic form $\omega$, can be reduced in a tubular neighbourhood of the orbit segment $\phi_{t} z, a \leq t \leq b$, to $\mathbb{R}^{2 n}$ with $\omega=\sum d x_{i} \wedge d y_{i}$, using the Darboux coordinate along $\phi_{t} z$.

Theorem 5.1. Let $d \phi_{t}$ denote the linearized flow of the Hamiltonian vector field $X_{H}$ on $\mathbb{R}^{2 n}$ and let $\lambda, \lambda^{*}$ denote Lagrange planes of $\mathbb{R}^{2 n}$, with $\alpha=$ vertical plane. Assume that $d \phi_{t} \lambda^{*} \cap \alpha=0, \quad 0 \leq t \leq b$, and

A1) $\partial^{2} / H \partial p^{2}>0$,

A2) $Q\left(\lambda, \alpha ; \lambda^{*}\right) \geq 0$,

A3) $\lambda \cap \alpha=0$.

Then $d \phi_{t} \lambda \cap \alpha=0, \quad 0 \leq t \leq b$.

Proof. Let $\Sigma_{\alpha}$ denote the singular cycle,

$$
\Sigma_{\alpha}=\{\gamma \in \Lambda(n) \mid \gamma \cap \alpha \neq 0\} .
$$

Using the fact that $\Lambda^{0}(\alpha)$ is connected, let $\gamma(s)$ denote a continuous curve, with $0 \leq s \leq 1$, in $\Lambda^{0}(\alpha)$, such that

$$
\gamma(0)=\lambda, \quad \gamma(1)=\lambda^{*} .
$$

Denote $\Gamma(s)=d \phi_{b} \gamma(1-s)$, and let $L_{b}^{*}, L_{b}$ denote the curves $d \phi_{t} \lambda^{*}, d \phi_{t} \lambda$ respectively, where $0 \leq t \leq b$.

Consider the closed curve of Lagrange planes $\Omega$, where

$$
\Omega=L_{b}^{-1} \circ \Gamma \circ \mathrm{E}_{b}^{*} \circ \gamma
$$

Our first observation is that $\Omega$ is contractible. Indeed, we define the curves $L_{a}$ and $L_{a}^{*}$ in the following way:

$$
L_{a}=d \phi_{t} \lambda, \quad L_{a}^{*}=d \phi_{t} \lambda^{*}, \quad 0 \leq t \leq a \leq b .
$$

Then, if $\Gamma_{a}=d \phi_{a} \gamma(1-s), 0 \leq s \leq 1$, it follows that

$$
\Omega_{0}=\gamma^{-1} \cdot \gamma \quad \text { (which is contractible), }
$$

and

$$
\Omega_{b}=\Omega
$$

Since $\Omega$ is contractible in $\Lambda(n)$, and $\pi_{1}(\Lambda(n))=\mathbb{Z}$ is Abelian, then

$$
[\Omega ; \alpha]=0 .
$$

Therefore, we may deduce that

$$
0=[\gamma ; \alpha]+\left[L_{b}^{*} ; \alpha\right]+[\Gamma ; \alpha]+\left[L_{b}^{-1} ; \alpha\right] .
$$

Moreover, since $[\gamma ; \alpha]=0$ by construction, and $\left[L_{b}^{*} ; \alpha\right]=0$ by assumption,

$$
[\Gamma ; \alpha]=-\left[L_{b}^{-1} ; \alpha\right]=[L ; \alpha] .
$$

In order to evaluate $[\Gamma ; \alpha]$, we use the Hörmander signature, as explained in Duistermaat [9] on p. 183,

$$
s\left(\gamma, \gamma^{\prime} ; \beta, \beta^{\prime}\right)=\frac{1}{2}\left\{\operatorname{sgn} Q\left(\gamma, \gamma^{\prime} ; \beta\right)-\operatorname{sgn}\left(\gamma, \gamma^{\prime} ; \beta^{\prime}\right)\right\} .
$$

This is the same as the Maslov index of a curve in $\Lambda^{0}(\gamma)$ from $\beta$ to $\beta^{\prime}$, followed by a curve in $\Lambda^{0}\left(\gamma^{\prime}\right)$ from $\beta^{\prime}$ back to $\beta$. 
To apply this formula for evaluation of $[\Gamma ; \alpha]$, we must check that

$$
\Gamma(0)=d \phi_{b} \lambda^{*} \quad \text { and } \quad \Gamma(1)=d \phi_{b} \lambda
$$

belong to $\Lambda^{0}\left(d \phi_{b} \alpha\right) \cap \Lambda^{0}(\alpha)$. By assumption, $\Gamma(0)$ and $\Gamma(1)$ belong to $\Lambda^{0}\left(d \phi_{b} \alpha\right)$. Without loss of generality, we may also assume that $\Gamma(0), \Gamma(1) \in \Lambda^{0}(\alpha)$. Indeed, if $d \phi_{b} \lambda \cap \alpha \neq 0$, then, by transversality, there is a small $\epsilon>0$ such that $d \phi_{b+\epsilon} \lambda \cap \alpha=0$, and $d \phi_{t} \lambda^{*} \cap \alpha=0$ for $0 \leq t \leq b+\epsilon$. In this case, the hypotheses of the theorem are fulfilled for the interval $0 \leq t \leq b+\epsilon$ and, as asserted,

$$
\Gamma(0), \Gamma(1) \in \Lambda^{0}\left(d \phi_{b} \alpha\right) \cap \Lambda^{0}(\alpha) .
$$

To continue the evaluation of $[\Gamma ; \alpha]$, assuming the transversality condition stated by the inclusion (18), we express $[\Gamma ; \alpha]$ using the Hörmander signature:

$$
[\Gamma ; \alpha]=\left[\Gamma ; d \phi_{b} \alpha\right]+s\left(d \phi_{b} \alpha, \alpha ; d \phi_{b} \lambda^{*}, d \phi_{b} \lambda\right) .
$$

We may deduce that

$$
\left[\Gamma ; d \phi_{b} \alpha\right]=\left[d \phi_{-b} \Gamma ; \alpha\right]=\left[\gamma^{-1} ; \alpha\right]=0 .
$$

Moreover,

$$
\begin{aligned}
s\left(d \phi_{b} \alpha, \alpha ; d \phi_{b} \lambda^{*}, d \phi_{b} \lambda\right) & =s\left(\alpha, d \phi_{-b} \alpha ; \lambda^{*}, \lambda\right) \\
& =-s\left(\lambda^{*}, \lambda ; \alpha, d \phi_{-b} \alpha\right) \quad(\text { see }[9] \mathrm{p} .184) \\
& =-\frac{1}{2}\left\{\operatorname{sgn} Q\left(\lambda^{*}, \lambda ; \alpha\right)-\operatorname{sgn} Q\left(\lambda^{*}, \lambda ; d \phi_{-b} \alpha\right)\right\} \\
& =\frac{1}{2}\left\{\operatorname{sgn} Q\left(\lambda, d \phi_{-b} \alpha ; \lambda^{*}\right)-\operatorname{sgn} Q\left(\lambda, \alpha ; \lambda^{*}\right)\right\} .
\end{aligned}
$$

In Lemma 2.1 we showed that $Q\left(d \phi_{-t} \alpha, \lambda^{*} ; \lambda\right)$ is a plus curve. By similarity, then, $Q\left(\lambda, d \phi_{-t} \alpha ; \lambda^{*}\right)$ is also a plus curve, and since $Q\left(\lambda, \alpha ; \lambda^{*}\right) \geq 0$ by hypothesis A2),

$$
\operatorname{sgn} Q\left(\lambda, d \phi_{-b} \alpha ; \lambda^{*}\right)-\operatorname{sgn} Q\left(\lambda, \alpha ; \lambda^{*}\right)=0 .
$$

Indeed, as the parameter $t$ moves from 0 to $b$, the eigenvalues of $Q\left(\lambda, d \phi_{-t} \alpha ; \lambda^{*}\right)$ can only cross zero from negative to positive. By virtue of A2), this implies that crossings cannot occur. Therefore, putting equations (19) and (20) together, we deduce that $[\Gamma ; \alpha]=0$, which, in turn, implies that $[L ; \alpha]=0$, as was to be shown.

\section{REFERENCES}

[1] W. Ambrose, The index theorem in Riemannian geometry, Annals of Mathematics, v. 73 (1961) 49-86 MR 24:A3608

[2] V. I. Arnold, On a characteristic class entering in quantization conditions, Funct. Anal. Appl, 1 (1967), 1-13 MR 35:2296

[3] V. Bangert, Closed geodesics on complete surfaces, Math. Ann. 251 (1980), 83-96 MR 81k:58027

[4] V. Bangert, Mather sets for twist maps and geodesics on tori, Dynamics Reported vol. 1, Teubner, Stuttgart, (1988), 1-56 MR 90a:58145

[5] V. Bangert, Minimal geodesics, Erg. Th. Dyn. Sys. 10 (1990), 263-286 MR 91j:58126

[6] G.D. Birkhoff, Dynamical Systems, Amer. Math. Soc. Colloq. Pub., v.9 (new ed.), Amer. Math. Soc., Providence, RI (1966) MR 35:1

[7] R. Bott, On the iteration of closed geodesics and the Sturm intersection theory, Comm. Pure Appl. Math. 9, (1956), 171-206 MR 19:859f

[8] R. Devaney, Blue sky catastrophes in reversible and Hamiltonian systems, Ind. Univ. Math. J., 26, (1977), 247-263 MR 55:4275 
[9] J.J. Duistermaat, On the Morse index in variational calculus, Adv. Math. 21, (1976), 173-195 MR 58:31190

[10] G.A. Hedlund, Geodesics on a two-dimensional Riemmanian manifold with periodic coefficients, Ann. of Math. 33, (1932), 719-739

[11] D. Kalish, The Morse index theorem where the ends are submanifolds, Trans. Amer. Math. Soc., 308, (1988), 341-348 MR 89i:58024

[12] W. Klingenberg, Riemmanian Geometry, De Gruyter Stud. Math. 1, Walter de Gruyter, Berlin, (1982) MR 84j:53001

[13] R.S. MacKay, private communication

[14] J.N. Mather, Existence of quasiperiodic orbits for twist homeomorphisms of the annulus, Topology 21, (1982), 457-467 MR 84g:58084

[15] J.N. Mather, Action minimizing invariant measures for positive Lagrangian systems, Math. Z. 207, (1991), 169-207 MR 92m:58048

[16] K. Meyer, Hamiltonian systems with a discrete symmetry, J. Diff. Eqns., 41, (1981), 228-238 MR 83a:58040

[17] D. Offin, A spectral theorem for reversible second order equations with periodic coefficients, Journal of Differential and Integral Equations, 5 (1992), 615-629 MR 93g:58027

[18] D. Offin, A Theorem on Uniform Hyperbolicity of Periodic and Limit Periodic Solutions of Planar Hamiltonian Systems, preprint, Queen's University Press (1998).

[19] H. Poincaré, Les méthodes nouvelles de la mécanique céleste, Vol. III, Gauthier-Villars, Paris, 1899; reprints, Dover, New York, 1957; Librairie Sci. Tech. Albert Blanchard, Paris, 1987. See p. 355. MR 19:414f; MR 89e:01054a

[20] H. E. Rauch, A contribution to differential geometry in the large, Ann. of Math. 54, (1951), 38-55 MR 13:159b

[21] F. W. Warner, Extension of the Rauch comparison theorem to submanifolds, Trans. Amer. Math. Soc., 122, (1966), pp. 341-356 MR 34:759]

Department of Mathematics and Statistics, Queen's University, Kingston, Ontario, CANADA K7L 3N6

E-mail address: offind@mast.queensu.ca 